\title{
Stochastic Dynamics of Quantum Physical Systems
}

\author{
Malkhaz Mumladze \\ Gori State University, Gori, Georgia \\ Email: mmumladze@mail.ru
}

How to cite this paper: Mumladze, M. (2016) Stochastic Dynamics of Quantum Physical Systems. Open Access Library Journal, 3: e3004.

http://dx.doi.org/10.4236/oalib.1103004

Received: August 25, 2016

Accepted: September 25, 2016

Published: September 28, 2016

Copyright $\odot 2016$ by author and Open Access Library Inc.

This work is licensed under the Creative Commons Attribution International

License (CC BY 4.0).

http://creativecommons.org/licenses/by/4.0/

\begin{abstract}
In this article we define and build the one method of description of stochastic evolution of a physical quantum system. For each quantum state $\omega \in E_{U}$ we construct the probability measure $\mu_{\omega}$ in the space $\left(P_{U}, S\right)$, where $P_{U}$ is the space of the pure states of the quantum system, $S$ the Borel $\sigma$-algebra in $P_{U}$. Farther, for any Hermit's positive element with norm $\|u\|=1$, in the $C^{*}$-algebra of observables $U$, we define the probability measure $\mu_{u}$ on the set of states $E_{U}$. If strongly continuous group $\left\{\alpha_{t}\right\}$ of ${ }^{*}$ automorphisms on $U$ describes the evolution of structure of observables, according to this, we have a picture of evolution of distribution of states of quantum system relatively to each observable $u$.
\end{abstract}

\section{Subject Areas}

Modern Physics

\section{Keywords}

Quantum Physical System, State, Observable, Probability Measure, Statistical Structure

\section{Introduction}

As known [1] a quantum physical system can be represented by a couple $(U, \mathfrak{I})$, where $U$ is some $C^{*}$-algebra which are hermit's elements that are called observables and some set $\mathfrak{I}$ of positive functional with norm one, called the quantum states of this physical system [2].

We say what the functional $\omega_{1}$ majorizes functional $\omega_{2}$ if $\omega_{1}-\omega_{2}$ is a positive functional [2].

The state $\omega$ quantum physical system is called the pure state if it majorizes only functional type $\lambda \omega, 0 \leq \lambda \leq 1$ [2]. Denote the set of all pure states on $C^{*}$-algebra $U$ 
by $P_{U}$.

In the set of all linear continuous functional on $C^{*}$-algebra $U$ we have topological structure, called as ${ }^{*}$ weakly topological structure [2] and defined by pre-basis:

$$
V\left(\omega, u_{1}, u_{2}, \cdots, u_{n}\right)=\left\{\omega^{\prime} \in U^{*}|| \omega\left(u_{i}\right)-\omega^{\prime}\left(u_{i}\right) \mid<\varepsilon, i=1,2, \cdots, n\right\},
$$

where $\omega, \omega^{\prime} \in U^{*}, u_{i} \in U$; according to this in the set $P_{U}$ we have the topological structure induced from this topological structure.

It is very known if $U$ commutative $C^{*}$-algebra, then every positive linear functional defines complex valued measure on the $P_{U}$, which is separable and locally compact space of pure states under the ${ }^{*}$ weekly topological structure. This measure is defined by corresponding $\mu \leftrightarrow F, F(f)=\int_{P_{U}} f(x) \mathrm{d} \mu$, for all $f \in P_{U}$ continuous function. In non-commutative case we cannot define the measure on $P_{U}$ in this way. In the work [3] for each linear functional, with norm one, we define the probability measure $P_{U}$ for commutative and non-commutative cases in other ways. It gives us the opportunity to present a quantum physical system as a statistical structure [4]. Representation of quantum physical system in this form, in our opinion, is more comfortable for the solution of problem of quantum system, for example for testing hypotheses [4]. In this paper, using this representation, we have tried to consider the dynamic of quantum physical system as a random process.

\section{Quantum Physical System as a Statistical Structure}

Denote by $\mathfrak{R}$ the set of Hermit's elements of $U \quad C^{*}$-algebra.

Easy to show that every linear functional on the $U \quad C^{*}$-algebra uniquely will be defined by its values on Banach subspace of Hermit's elements,as it's known [2] that every element $u$ of $C^{*}$-algebra $U$ uniquely represented as $u_{1}+i u_{2}$, where $u_{1}$ and $u_{2}$ are Hermit's.

Every a Hermit's element $u \in \mathfrak{R}$ in the $C^{*}$-algebra $U$ any can be represented by integral

$$
u=\int_{-\infty}^{\infty} \lambda \mathrm{d} p_{\lambda}^{u}
$$

where $\left\{p_{\lambda}^{u}\right\}_{\lambda \in R}\left(p_{\lambda}^{u}\right)^{2}=p_{\lambda}^{u}$ projectors and represents the partition of unityof Hermit's element $u \in \mathfrak{R}$ [5].

Correspond to projector $p_{\alpha}^{2}=p_{\alpha} \in U$ the family $\left\{p_{\lambda}^{p_{\alpha}}\right\}_{\lambda \in R}$ of elements of the $C^{*}$ algebra $U$ which has the condition: $p_{\lambda}^{p_{\alpha}}=0$, if $\lambda<1, p_{\lambda}^{p_{\alpha}}=p_{\alpha}$, if $\lambda=1$ and $p_{\lambda}^{p_{\alpha}}=E$, if $\lambda>1$, where $E$ is the unit element in the algebra $U$. It is clear that.

$$
p_{\alpha}=\int_{-\infty}^{\infty} \lambda \mathrm{d} p_{\lambda}^{p_{\alpha}}
$$

If $u \in U$, then $u=u_{1}+i u_{2}$, where $u_{1}$ and $u_{2}$ are Hermit's elements. The representation such $u$ will be 


$$
u=u_{1}+i u_{2}=\int_{-\infty}^{\infty} \lambda \mathrm{d} p_{\lambda}^{u_{1}}+i \int_{-\infty}^{\infty} \lambda \mathrm{d} p_{\lambda}^{u_{1}}
$$

Obviously, if $\omega$ some linear continuous functional on $C^{*}$-algebra $U$, then from the last equality we will have

$$
\omega(u)=\omega\left(u_{1}+i u_{2}\right)=\omega\left(\int_{-\infty}^{\infty} \lambda \mathrm{d} p_{\lambda}^{u_{1}}\right)+\omega\left(\int_{-\infty}^{\infty} \lambda \mathrm{d} p_{\lambda}^{u_{2}}\right)=\int_{-\infty}^{\infty} \lambda \mathrm{d} \omega\left(p_{\lambda}^{u_{1}}\right)+i \int_{-\infty}^{\infty} \lambda \mathrm{d} \omega\left(p_{\lambda}^{u_{2}}\right),
$$

where $u_{1}$ and $u_{2}$ are Hermit's elements.

Let, $\left\{p_{\alpha}\right\}$ be the set of all one dimensional projectors on $C^{*}$-algebra $U$ and $\omega$ pure state, then the last equality follows that this state has the non zero meaning only on some projector $p_{\alpha} \in\left\{p_{\alpha}\right\}$ and the meaning 0 on the other one dimensional projectors. Otherwise, we can always construct such functional which will not have the type $\lambda \omega, 0 \leq \lambda \leq 1$ and well majorized by the pure state $\omega$. So how, if functional $\omega$ is a pure state, then $\omega\left(u^{2}\right) \geq 0$ for all Hermit's elements $u$.

Let, $\omega\left(p_{\alpha^{\prime}}\right) \neq 0$ and $\omega_{\alpha^{\prime}}$ such functional, which has the non zero meaning $\varepsilon$ only on some projector $p_{\alpha^{\prime}} \in\left\{p_{\alpha}\right\}$ and the meaning 0 on the other one dimensional projectors. It is clear, if we take $\varepsilon$ sufficiently small, thenwe can achieve, that for every $u \in \Re$ will have place inequality

$$
\left(\omega-\omega_{\alpha^{\prime}}\right)\left(u^{2}\right)=\int_{-\infty}^{\infty} \lambda^{2} \mathrm{~d}\left(\omega-\omega_{\alpha^{\prime}}\right)\left(p_{\lambda}^{u}\right) \geq 0 .
$$

It means, that the pure state $\omega$ majorize the functional $\omega_{\alpha^{\prime}}$ which does not have the type $\lambda \omega, 0 \leq \lambda \leq 1$, but this is impossible. It follows that the pure states are sach functional which satisfy the condition $\omega_{\alpha}\left(p_{\beta}\right)=\delta^{\alpha \beta}$.

An integral representation of Hermit's elements follows that for the pure states $\omega_{\alpha}$ has a place of equality $\omega_{\alpha}(u)=\lambda_{\alpha}^{u}$, where $\lambda_{\alpha}^{u}$ is some element of spectrum of Hermit element $u \in \mathfrak{R}$. It gives opportunity to identify every pre state with the set of number $\left\{\lambda_{\alpha}^{u}\right\}_{u \in \Re}$, where $p_{\alpha}(u)=\lambda_{\alpha}^{u}$.

Consider the Tikhonov's product $\Sigma=\underset{u \in \Re}{\otimes} \sigma_{u}$, where $\sigma_{u} \subset R$ spectrum of element $u \in \Re$.

It is clear, $P_{U} \subset \Sigma$, because $P_{U}$ the set of such elements in product $\Sigma=\underset{u \in \Re}{\otimes} \sigma_{u}$ which represents linear continue maps with respect to the topological structure in $\Re$ which is defined by the norm:

$$
P_{U}=\left\{\omega_{\alpha}: \Re \rightarrow \bigcup_{u \in \Re} \sigma_{u} \mid \omega_{\alpha}(u)=\lambda_{\alpha}^{u}, \omega_{\alpha}\left(k_{1} u_{1}+k_{2} u_{2}\right)=k_{1} \omega_{\alpha}\left(u_{1}\right)+k_{2} \omega_{\alpha}\left(u_{2}\right)\right\} .
$$

Consequently in the set $P_{U}$ we have $\Sigma=\otimes_{u \in \Re} \sigma_{u}$, induced from Tikhonov's product $\Sigma=\underset{u \in \Re}{\otimes} \sigma_{u}$ topological structure. This topological structure coincide with theinduced topological structure from ${ }^{*}$ weakly topological structure on set of functionals on $C^{*}$ algebra $U$.

We can also identify the set $P_{U}$ with the set of one dimensional projectors $\left\{p_{\alpha}\right\}$. We call $P_{U}$ as physical space of quantum system.

In the work [3] we have proved: 
Theorem 1. Every state $\omega \in \mathfrak{I}$ in space $P_{U}$ with ${ }^{*}$ weakly topological structure, defined on the Borel $\sigma$-algebra of a probability measure $\mu_{\omega}$.

This measure constructed as: for the subset $\left\{p_{\alpha_{\beta}}\right\}$ of $P_{U}$, measure $\mu_{\omega}\left(\left\{p_{\alpha_{\beta}}\right\}\right)$ is norm of positive functional $v,\|v\| \leq 1$, if this exists, which values on the elements of this subset are coincide to corresponding to values of the state $\omega$. i.e. $\mu_{\omega}\left(\left\{p_{\alpha_{\beta}}\right\}\right)=\|v\|$.

Every measure $\mu_{\omega}$ describes distribution elementary particle in physical space of quantum system $P_{U}$ in the state $\omega$.

If $C^{*}$-algebra $U$ has a unit, then in the space $U^{*}$ with ${ }^{*}$ weakly topological structure the set of all state $\mathfrak{I}$ is convex compact set and represent convex linear combination of pure states $\zeta_{1}, \zeta_{2}, \cdots, \zeta_{n}$ from the set $P_{U}$ :

$$
\omega=k_{1} \zeta_{1}+k_{2} \zeta_{2}+\cdots+k_{n} \zeta_{n}, k_{i} \geq 0, \sum_{i=1}^{n} k_{i}=1
$$

or limit of sequence $\omega_{1}, \omega_{2}, \cdots, \omega_{1}, \cdots$, where

$\omega_{l}=k_{1}^{l} \zeta_{1}^{l}+k_{2}^{l} \zeta_{2}^{l}+\cdots+k_{n_{l}}^{l} \zeta_{n_{l}}^{l}, k_{i}^{l} \geq 0, \sum_{i=1}^{n_{l}} k_{i}^{l}=1 \quad$ [2]

This means, that elements of set $P_{U}$ are the extreme points of set [2].

Because each state $\omega \in \mathfrak{I}_{u}$ defines a probability measure $\mu_{\omega}$ on couple $\left(P_{U}, S\right)$, where $S$ is borel $C^{*}$-algebra therefore it is easy to show, that every $\mu_{\omega}$ represent convex linear combination

$$
\mu_{\omega}=\sum_{i=1}^{n} k_{i} \mu_{\varsigma_{i}}, k_{i} \geq 0, \sum_{i=1}^{n} k_{i}=1
$$

of Dirak measures $\mu_{\varsigma_{i}}$ where $\xi_{i} \in P_{U}, i=1,2, \cdots, n$ or limit of sequense $\left\{\mu_{\omega_{n}}\right\}_{n \in N}$, where $\mu_{\omega_{n}}=k_{1}^{n} \mu_{\varsigma_{1}^{n}}+k_{2}^{n} \mu_{\varsigma_{2}^{n}}+\cdots+k_{n_{l l}}^{n} \mu_{\varsigma_{l}^{n}}, k_{l}^{n} \geq 0, \sum_{i=1}^{l} k_{i}^{n}=1 \quad$ [2]

For every state $\omega \in \mathfrak{I}$ we have $\int_{-\infty}^{\infty} \mathrm{d} \omega\left(E_{\lambda}^{u}\right)=1$ therefore it is easy that the value of quantum state on observable $u \in \Re$ is the middle value of this observable. The value $\omega(u) \in R$ is called the middle value of observable $u \in \mathfrak{R}$ of quantum physical system in the state $\omega \in \mathfrak{I}$.

All told above follows that a quantum physical system is an object, so-called statistical structure [4]:

$$
\left(U, P_{U}, \mathfrak{I}, S,\left\{\mu_{\omega}\right\}, \omega \in \mathfrak{I}\right),
$$

where $U$ some $C^{*}$-algebra, Hermit element of which are called observables of this system, $P_{U}$ is the space of quantum system, $S$ Borel $\sigma$-algebra in $P_{U}, \mu_{\omega}$ the probability measure defined by state $\omega \in \mathfrak{J}$ and which describes distribution elementary particle in physical space of quantum system $P_{U}$ in the state $\omega$.

\section{A Stochastic Dynamics of Quantum System}

Theorem 2. Every Hermit's element $u,\|u\|=1$ in $\sigma$-algebra $U$ defines probability measure on the set of states $E_{U}$. 
Proof It is well-known that the map $\pi: U \rightarrow U^{* *}$ defined by formula $\pi(u)(\omega)=F_{u}(\omega)=\omega(u)$ is isometric embedding $U$ as Banach space in the double conjugate space $U^{* *}$ [3]. If $\omega$ is a state then $\omega\left(u^{*} u\right) \geq 0$ [2]; it follows that if $u \in U$ is positive element, then $u=v^{*} v, v \in U$ and $\omega(u) \geq 0$.

Thus if $u \in U$ is positive element then $F_{u}(\omega) \geq 0$ for each state on $U$. Because $\pi$ is isometric, and therefore $\left\|F_{u}\right\|=\|u\|$.

$$
\left(F_{u}+F_{v}\right)(\omega)=F_{u}(\omega)+F_{v}(\omega)=\omega(u)+\omega(v)=\omega(u+v)=F_{u+v}(\omega) .
$$

If $u$ is hermit's element $u=\int_{-\infty}^{\infty} \lambda \mathrm{d} p_{\lambda}^{u}$, because, for such elements $F_{u}=\int_{-\infty}^{\infty} \lambda \mathrm{d} F_{p_{\lambda}^{u}}$ and

$$
F_{u}(f)=\int_{-\infty}^{\infty} \lambda \mathrm{d} F_{p_{\lambda}^{u}}(\omega)=\int_{-\infty}^{\infty} \lambda \mathrm{d} \omega\left(p_{\lambda}^{u}\right) .
$$

Let $u,\|u\|=1$ be hermit's positive element in $U$, then spectrum $\sigma_{u} \subset[0,1]$. Let $E_{U}$ be the set of all states on $U$, if $O \subset E_{U}$ is a set of states; we assume the measure $\mu_{u}(O)$ of this set is $\lambda$, if $O$ consists for all such element $\omega$ for which $\omega(u) \leq \lambda$, $\lambda \in \sigma_{u}, \sup _{\omega}\{\omega(u)\}=\lambda$. Since $\sigma_{u} \subset[0,1], 0$ and 1 are elements of $\sigma_{u}$ [5], and therefore $\mu_{u}\left(E_{U}\right)=1$. It is clear that, if $\lambda_{1}, \lambda_{2} \in \sigma_{u}, \lambda_{1}<\lambda_{2}$ then

$$
\left\{f \mid \omega(u) \leq \lambda_{1}\right\} \subset\left\{\omega \mid \omega(u) \leq \lambda_{2}\right\} .
$$

If we assume $\mu_{u}\left(\left\{\omega \mid \omega(u) \leq \lambda_{2}\right\} \backslash\left\{\omega \mid \omega(u) \leq \lambda_{1}\right\}\right)=\lambda_{2}-\lambda_{1}$, then we get a measure on $E_{U}$.

The sets for which we define measure, make $\sigma$-algebra in $E_{U}$. This is not a Borel's $\sigma$-algebra in space $E_{U}$ whit the ${ }^{*}$ weekly topology. Denote it by $S_{u}^{*}$. Thus, we define on $\left(E_{U}, S_{u}^{*}\right)$ probability measure. The theorem is proved.

Consider the family of measures $\left\{E_{U}, S_{u}^{*}, \mu_{u}, u \in \mathfrak{R}_{1}^{+}\right\}$defined above, where $\mathfrak{R}_{1}^{+}$is the set of positive hermit's elements whit norm $1, S_{u}^{*}$ is corresponding to hermit's element $u \in \mathfrak{R}_{1}^{+}, \sigma$-algebra in $E_{U}$.

Let $\left(U, P_{U}, \mathfrak{I}, S,\left\{\mu_{\omega}\right\}, \omega \in \mathfrak{I}\right)$ statistical structure represent a quantum physical system, $\mathfrak{I} \subset E_{U}$. For each $u \in \mathfrak{R}_{1}^{+}$we can define the measure $\mu_{\mathfrak{I}}^{u}$ on the set of states $\mathfrak{I}$ of given quantum physical system such:

$$
\mu_{\mathfrak{I}}^{u}(O)=\lambda \text { if } O \subset \mathfrak{I}, O=\mathfrak{I} \cap \mu_{u}^{-1}(\lambda) .
$$

Literally, we have defined measure $\mu_{\mathfrak{I}}^{u}$ on the set of measures $\left\{\mu_{\omega}\right\}_{\omega \in \mathfrak{I}}$, of which each element $\mu_{\omega}$ describes distribution elementary particles in physical space $P_{U}$ of quantum system in the state $\omega$.

If $\left\{a_{t}\right\}_{t \in R}$ is strongly one parametric group of maps of $C^{*}$-algebra $U$ whit unity and $a_{t}(1)=1$ for all $t \in R$, then following conditions are equivalent [2]:

1) All $a_{t}^{*}$ automorphisms of $U$;

2) $\left\|a_{t}\right\| \leq 1$ for all $t \in R$;

3) $a_{t}\left(U_{+}\right) \subset U_{+}$, where $U_{+}$is the set of positive elements in $U$; 
4) $a_{t}^{*}\left(E_{U}\right) \subset E_{U}$, for all $t \in R$.

Each defined measure $\mu_{\mathfrak{I}}^{u}$ describes distribution of states in $\mathfrak{I}$ relatively to middle value of observable $u$ over states in $\mathfrak{I}$, or distribution of elementary particles in physical space $P_{U}$ of quantum system in the states $\omega \in \mathfrak{I}$ relatively to middle value of observable $u$ over states in $\mathfrak{I}$.

It follows: If strongly continuous one parametric group of automorphisms $\left\{a_{t}\right\}_{t \in R}$ describes dynamic of structure of observables, according to this, we have a picture of evolution of distribution of states quantum system $\left(U, P_{U}, \mathfrak{I}, S,\left\{\mu_{\omega}\right\}, \omega \in \mathfrak{I}\right)$ relatively to each observable $u$.

Such, the representation of quantum physical system as a statistical structure allows formalizing the dynamics of the quantum system as a random process.

\section{References}

[1] Bogolyubov, H.H., Logunov, A.A., Oksak, A.I. and Todorov, I.T. (1987) General Principles of the Quantum Field Theories. Nauka, Moscow. (In Russian)

[2] Bratteli, O. and Robinson, D. (1982) Operator Algebras and Quantum Statistical Mechanics. Nauka, Moscow. (In Russian)

[3] Mumladze, M. and Zerakidze, Z. (2015) The Criteria of Checking Hypotheses of Quantum States of Quantum Physical System. Journal of Algebra, Number Theory. Advances and Applications, 14, 57-67.

[4] Zerakidze, Z. and Mumladze, M. (2015) Statistical Structures and Consistent Criteria for Checking Hypotheses. LAMBERT Academic Publishing, Saarbrucken.

[5] Liusterniik, L.A. and Sobolev, V.I. (1965) Elements of Functional Analyses. Nauka, Moscow. (In Russian)

Submit or recommend next manuscript to OALib Journal and we will provide best service for you:

- Publication frequency: Monthly

- 9 subject areas of science, technology and medicine

- Fair and rigorous peer-review system

- Fast publication process

- Article promotion in various social networking sites (LinkedIn, Facebook, Twitter, etc.)

- Maximum dissemination of your research work

Submit Your Paper Online: Click Here to Submit

Or Contact service@oalib.com 